\title{
BCREval: a computational method to estimate the bisulfite conversion ratio in WGBS
}

\author{
Junhua Zhou', Minqiong Zhao', Zefang Sun', Feilong Wu', Yucong Liu', Xianghua Liu², Zuping He', \\ Quanze $\mathrm{He}^{3^{*}}$ and Quanyuan $\mathrm{He}^{1 *}$ (i)
}

\begin{abstract}
Background: Whole genome bisulfite sequencing (WGBS) also known as BS-seq has been widely used to measure the methylation of whole genome at single-base resolution. One of the key steps in the assay is converting unmethylated cytosines into thymines (BS conversion). Incomplete conversion of unmethylated cytosines can introduce false positive methylation call. Developing a quick method to evaluate bisulfite conversion ratio (BCR) is benefit for both quality control and data analysis of WGBS.

Results: Here we provide a computational method named "BCREval" to estimate the unconverted rate (UCR) by using telomeric repetitive DNA as native spike-in control. We tested the method by using public WGBS data and found that it is very stable and most of BS conversion assays can achieve> 99.5\% efficiency. The non-CpG DNA methylation at telomere fits a binomial model and may result from a random process with very low possibility (the ratio < 0.4\%). And the comparison between BCREval and Bismark (Krueger and Andrews, Bioinformatics 27:1571$1572,2011)$, a widely used BCR evaluator, suggests that our algorithm is much faster and more efficient than the latter.

Conclusion: Our method is a simple but robust method to QC and speculates BCR for WGBS experiments to make sure it achieves acceptable level. It is faster and more efficient than current tools and can be easily integrated into presented WGBS pipelines.
\end{abstract}

Keywords: DNA methylation, Whole genome bisulfite sequencing (WGBS), Bisulfite conversion ratio (BCR), Telomere

\section{Background}

DNA methylation, as a fundamental epigenetics modification, plays critical roles in various biology processes including embryonic development, pluripotency maintenance, genomic imprinting, gene expression regulation, and genomic stability maintenance. It involves the addition of a methyl group to the carbon 5 position of CpG (most of tissues) and non-CpG dinucleotides (embryonic stem cell et.al.) by DNA methyltransferases in a tissue-specific way. Knowledge of the genomic methylation landscape is essential for understanding how

\footnotetext{
* Correspondence: hqyone@hunnu.edu.cn; hqzlul@163.com

'School of Medicine, Hunan Normal University, Tongzipo Road 371,

Changsha 410013, People's Republic of China

${ }^{3}$ The Affiliated Suzhou Hospital of Nanjing Medical University, Suzhou,

People's Republic of China

Full list of author information is available at the end of the article
}

methylation patterns are established and maintained and the significance of DNA methylation in development and disease.

Several methods exist for measuring DNA methylation in genomic wide including Whole genome bisulfite sequencing (WGBS), reduced representation bisulfite sequencing (RRBS), post-bisulfite adapter tagging (PBAT) [1] and Methylated DNA immunoprecipitation based methods (MeDIP-chip and MeDIP-seq) [2]. In which, WGBS is the only one to identify all the $\mathrm{C}$ information and global pattern therefore has become the standard profiling method in major epigenome consortiums such as NIH Roadmap [3], ENCODE [4], Blueprint [5] and IHEC [6]. In this assay, genomic DNA is purified and sheared into fragments and then treated with bisulfite, a chemical that converts unmethylated cytosine but not

(c) The Author(s). 2020 Open Access This article is distributed under the terms of the Creative Commons Attribution 4.0 International License (http://creativecommons.org/licenses/by/4.0/), which permits unrestricted use, distribution, and reproduction in any medium, provided you give appropriate credit to the original author(s) and the source, provide a link to the Creative Commons license, and indicate if changes were made. The Creative Commons Public Domain Dedication waiver (http://creativecommons.org/publicdomain/zero/1.0/) applies to the data made available in this article, unless otherwise stated. 


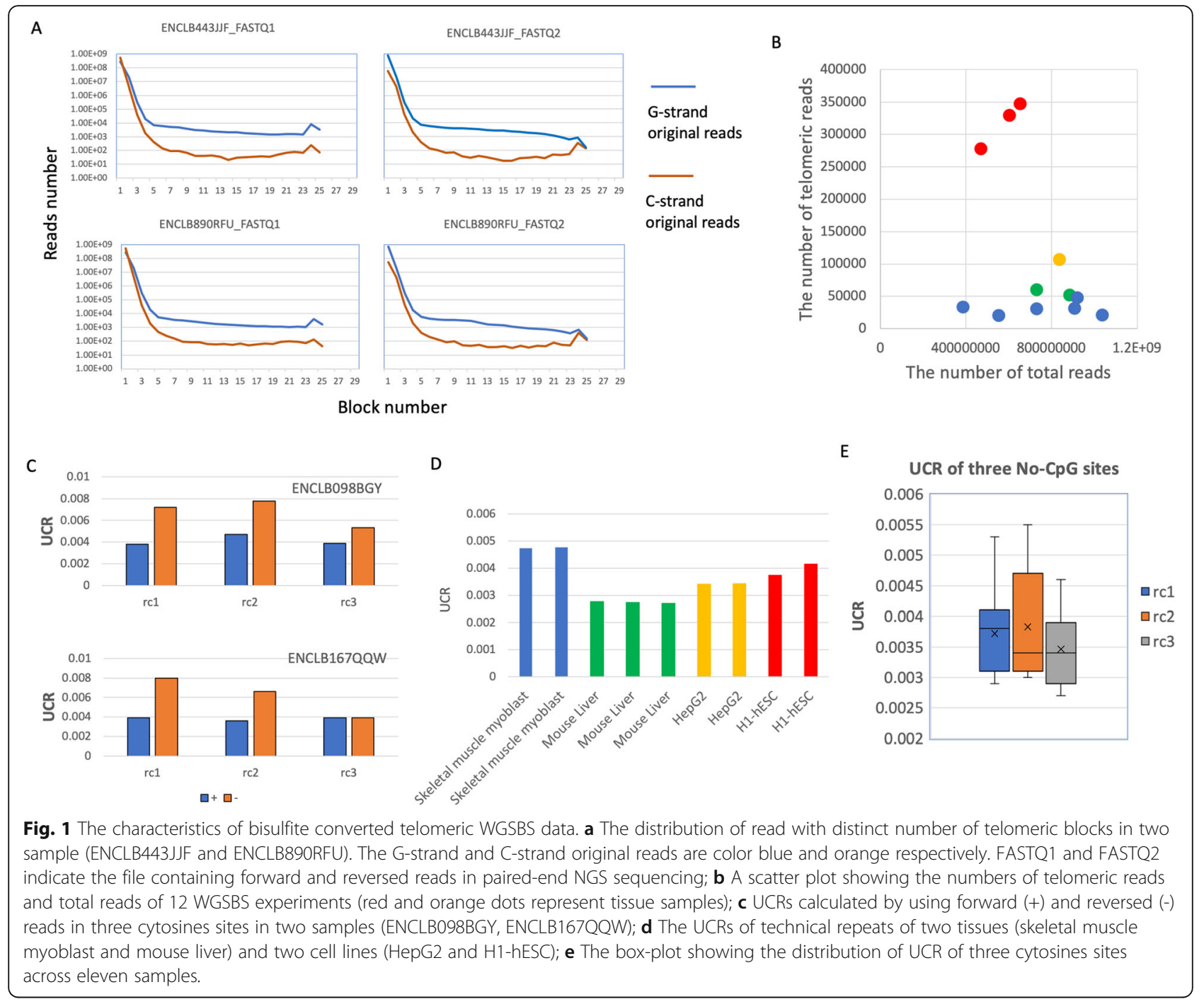

methylated ones to uracil. The bisulfite converted genomic DNA is then sequenced by a Next-generation sequencing platform. The methylation states of cytosines are determined by searching T-C mismatches between sequences obtained and the reference genome.

In a WGBS assay, it was implicitly assumed that this bisulfite conversion should run to completion. However, it is not always the case. Incomplete conversion of unmethylated cytosines will make it impossible to distinguish unconverted unmethylated cytosines from methylated ones and therefore will result in false positive methylation calls $^{7}$. At other hand, prolonged bisulfite treatment causes DNA degradation in a way of remaining methylated reads. So it is important to estimate bisulfite conversion ratio (BCR) for each WGBS experiment.

Some studies have used observed BCR in closed non-CpG sites to estimate $\mathrm{BCR}$ by assuming very low methylation ratio of non-CpG sites which may not be the case especially in some cell type (e.g. ES cell). Additionally, some (C/T) SNPs and low coverage regions may also introduce noise for the strategy. Another option to estimate BCR is using spike-in control of nonnative DNA with a known methylation state, which increases the complexity of procedure and rely on another assumption that DNA and spike DNA have the same conversion properties which is also questionable.

Telomeres are distinctive structures found at the ends of chromosomes, which protect the ends of chromosomes from deterioration or fusion with neighboring chromosomes. In vertebrate telomeres, the sequence of TTAGGG is repeated strictly approximate 3000 times and can reach up to 15,000 base pairs in length. Its complementary DNA strand contains CCCTAA repeats which have three non-CpG sites (one $\mathrm{CpT}$ and two $\mathrm{CpC}$ sites) for each repeat. As there are a lot of non-CpG site exist in telomeres, using telomeric DNA as native spikein control may be a better way to evaluate BCR.

Here, we provide a computational method named "BCREval" to evaluate BCR using telomeric DNA as 


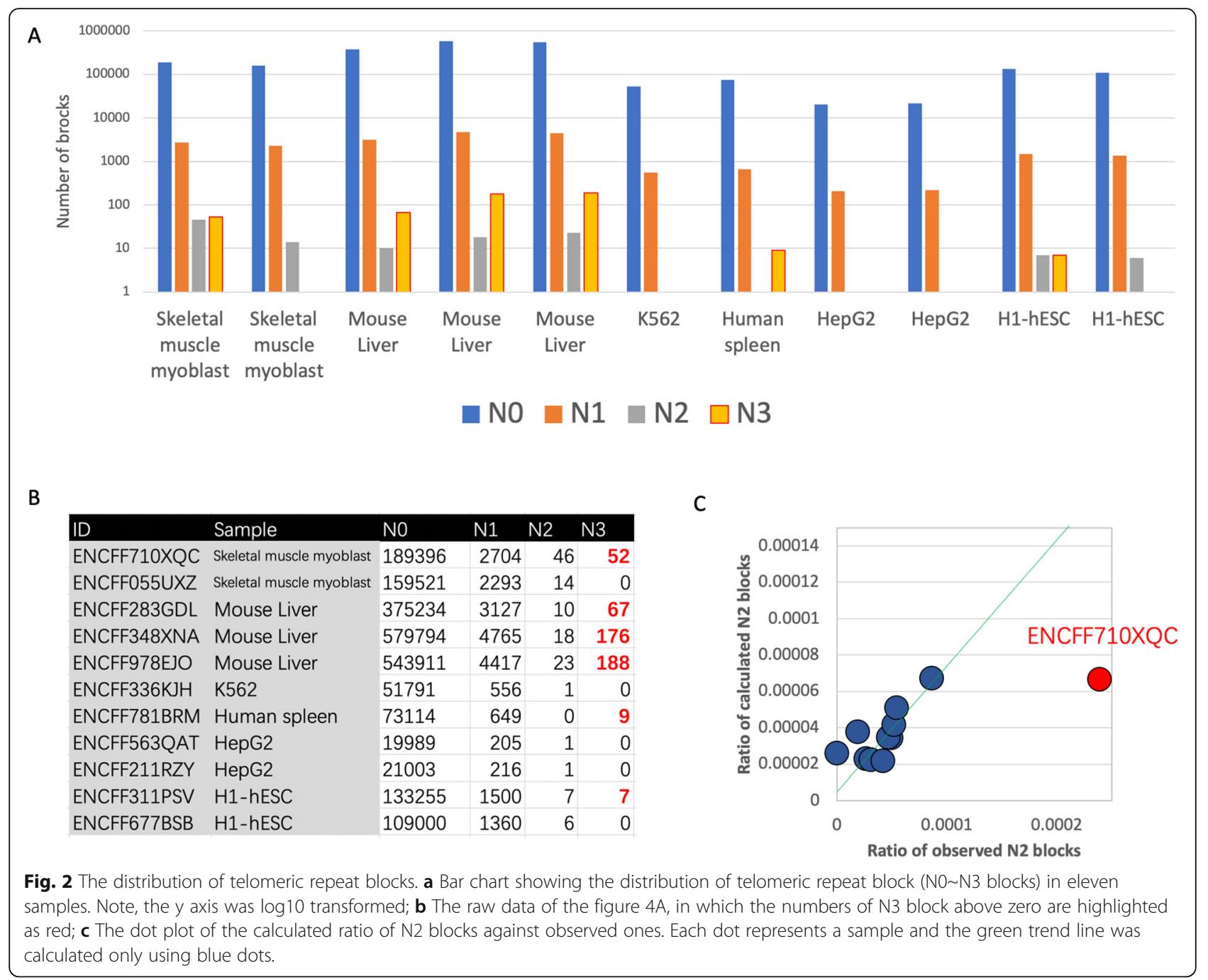

native spike-in control for WGBS experiment. We tested the method using 11 public available WGBS data of various cell lines and tissues and found that this method is easy to use and stable. In most of cases, the BCR of telomeric DNA is above $99.5 \%$ suggesting that telomeric DNA are unmethylated in all test samples and the DNA methylation at telomere fits a binomial model and may result from a random process with very low possibility. Interestedly, no significant differences of non-CpG methylation ratio was found in three non-CpG cytosine sites. Additionally, the comparison between BCREval and Bismark [8], a widely used BCR evaluator, shows that our algorithm is much faster and more efficient than the latter. Finally, the python script implementing the method is ready and easy to integrate into presented WGBS pipelines.

\section{Results}

We counted the number of reads with $\mathrm{n}$ repeated blocks ( $\mathrm{n}$ range from 1 to 30 ) in both forward and reversed FASTQ files (Fig. 1a). As expected, along with the increasing of n, the number of matched reads decreases dramatically and then becomes stable after $n>=8$ (Fig. 1a). Therefore we used 8 as the minimal repeated block number to distinguish telomeric reads from others. It is consistence with the fact that 58 of 59 (TTAGGG) locate on either subtelomeric or telomeric regions in human genome (hg38) (Additional file 1: Figure S1). The peaks at $n=24 \sim 25$ indicates that many reads are composed by telomeric repeats completely and suggests the ability of WGBS to capture telomeric DNA. Interestedly, the data also show that the $\mathrm{G}$ strand original telomeric reads (50\% GC contents) are much more than ones from C strand (low GC contents) in both FASTQ files, which may result in the GC bias of PCR step (Fig. 1a). Interestedly, comparing with cell-lines, all tissue samples have higher ratios of telomeric reads to total ones (Fig. 1b).

The unconverted ratio (UCR) of three non-CpG sites of telomeric reads were calculated for both forward and reversed FASTQ files. We found that UCRs from reversed FASTQs file frequently slightly higher than their 
count parts from forward FASTQs (Fig. 1c). The possible reason is that all reversed telomeric reads were always sequenced from telomeres to centromeres which may introduce false telomeric blocks at the end of reads. Therefore, we only used forward FASTQ files for further analyses. We also found that the variants of UCRs among technical replicates are very small suggesting good reliability of the method (Fig. 1d).

Methylated cytosines are found primarily at $\mathrm{CpG}$ dinucleotides, but are also found at non-CpG sites (CpA, $\mathrm{CpT}$ and $\mathrm{CpC}$ ) in specific mammal cell types including pluripotent stem cells, oocytes, neurons, and glial cells [7]. Using our method, we found that in most cases, the unconverted cytosines in telomere are rare $(\mathrm{UCR}<0.5 \%)$ and no significant difference of UCRs were found among three non-CpG sites (Fig. 1e), suggesting the bisulfite conversion treatment is very realizable and has high efficiency (>99.5\%). However, we do observe that $1.1 \%$ $1.7 \%$ telomeric blocks have unconverted cytosines in all samples, which may result from either the failure of bisulfite conversion or cytosine methylation. The UCRs of telomere blocks were solved using the formula 2 and then R2s and R3s were calculated using formula 3 and 4 respectively. As the data shown, with the exception of ENCFF710XQC, there is a good positive correlation between observed and calculated R2s(Fig. 2a,b) and their paired-values are comparable (Fig. 2c), which suggests that the binomial model fits these data well and the cytosine methylation are non-specific and random events in these samples.

Based on the random model, the expected number of R3 blocks should be zero for all samples (Additional file 1: Table S1). However, there are six of eleven samples have N3 blocks, which may result from either untreated genomic

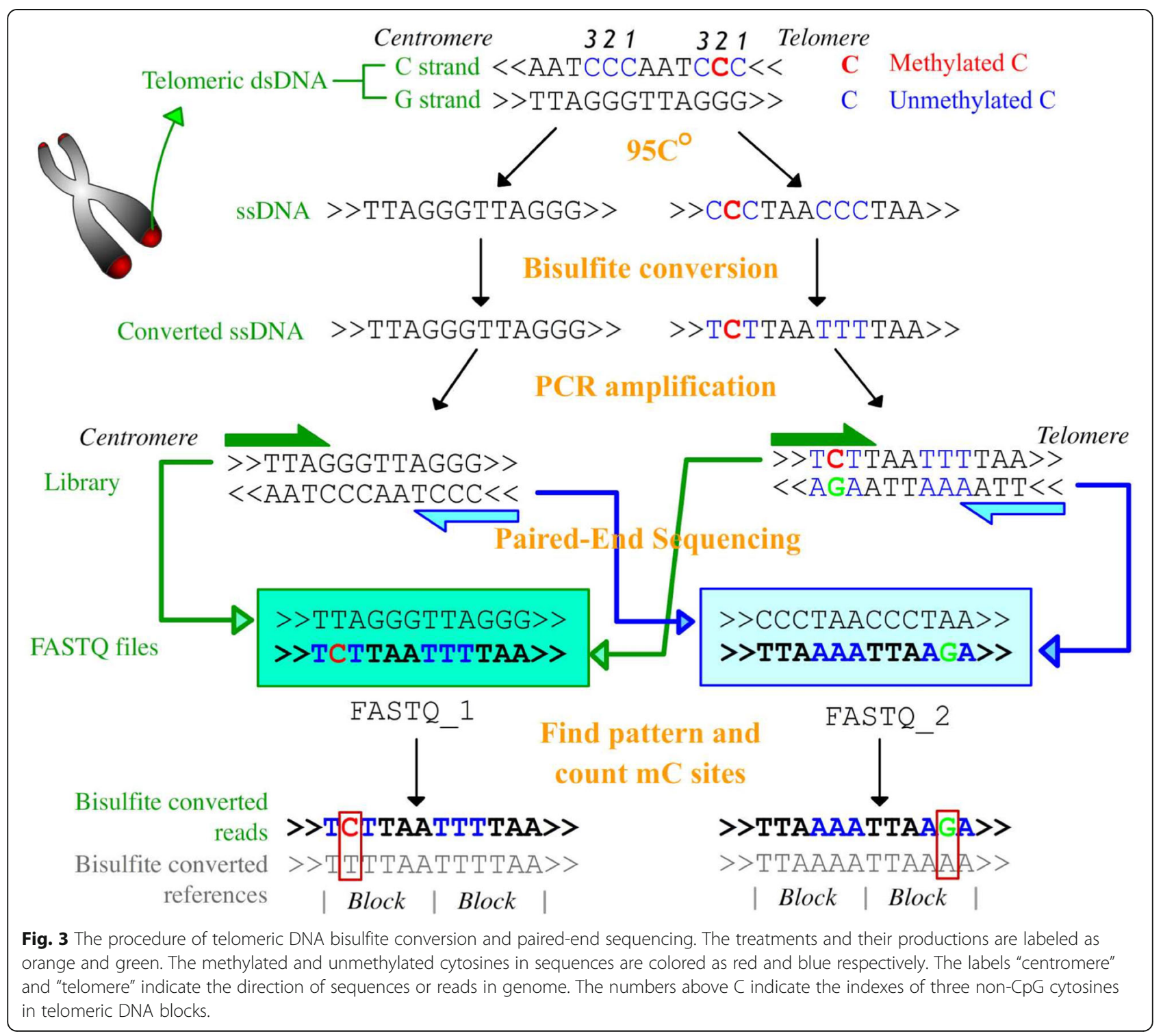


Table 1 The performance comparison of BCREval and Bismark

\begin{tabular}{|c|c|c|c|c|c|c|c|c|}
\hline \multirow[t]{2}{*}{ ENCODE ID } & \multirow{2}{*}{$\begin{array}{l}\text { File } \\
\text { Size }\end{array}$} & \multirow{2}{*}{$\begin{array}{l}\text { Reads } \\
\text { Number }\end{array}$} & \multicolumn{2}{|c|}{ Processing Time } & \multicolumn{2}{|c|}{ Memory Usage } & \multicolumn{2}{|c|}{$\mathrm{CHH}$ methylation ratio \% } \\
\hline & & & Bismark & BCREval & Bismark & BCREval & Bismark & BCREval \\
\hline ENCFF055UXZ & $1.1 \mathrm{G}$ & $12 \mathrm{M}$ & $4 \mathrm{~h} 49 \mathrm{~m}$ & $10 \mathrm{~m}$ & $10 \mathrm{G}$ & $44 \mathrm{M}$ & 0.7 & 0.56 \\
\hline ENCFF336KJH & $687 \mathrm{M}$ & $12 \mathrm{M}$ & $4 \mathrm{~h} 8 \mathrm{~m}$ & $9 m$ & $10 \mathrm{G}$ & $44 \mathrm{M}$ & 0.5 & 0.54 \\
\hline ENCFF677BSB & $926 \mathrm{M}$ & $12 \mathrm{M}$ & $5 \mathrm{~h} 28 \mathrm{~m}$ & $9 m$ & $10 \mathrm{G}$ & $44 \mathrm{M}$ & 1.1 & 0.42 \\
\hline ENCFF781BRM & $833 \mathrm{M}$ & $12 \mathrm{M}$ & $5 \mathrm{~h} 5 \mathrm{~m}$ & $9 m$ & $10 \mathrm{G}$ & $44 \mathrm{M}$ & 0.5 & 0.26 \\
\hline ENCFF710XQC & $1011 \mathrm{M}$ & $12 \mathrm{M}$ & $5 \mathrm{~h} 5 \mathrm{~m}$ & $10 \mathrm{~m}$ & $10 \mathrm{G}$ & $44 \mathrm{M}$ & 0.8 & 0.45 \\
\hline ENCFF211RZY & $1.1 \mathrm{G}$ & $12 \mathrm{M}$ & $4 \mathrm{~h} 39 \mathrm{~m}$ & $10 \mathrm{~m}$ & $10 \mathrm{G}$ & $44 \mathrm{M}$ & 0.5 & 0.17 \\
\hline ENCFF563QAT & $821 \mathrm{M}$ & $12 \mathrm{M}$ & $4 \mathrm{~h} 31 \mathrm{~m}$ & $8 \mathrm{~m}$ & $10 \mathrm{G}$ & $44 \mathrm{M}$ & 0.5 & 0.18 \\
\hline ENCFF311PSV & $686 \mathrm{M}$ & $12 \mathrm{M}$ & $4 \mathrm{~h} 36 \mathrm{~m}$ & $10 \mathrm{~m}$ & $10 \mathrm{G}$ & $44 \mathrm{M}$ & 1.1 & 0.3 \\
\hline
\end{tabular}

DNA contamination or enzymatic methylation. It is easy to distinguish them using distribution patterns, because the N3 blocks from contamination usually cluster together and enzymatic methylated ones should be dispersed. As the data shown that all N3 blocks from four samples (ENCFF710XQC, ENCFF283GDL, ENCFF348XNA, ENCFF978EJO) cluster into several N3 block richen reads suggesting untreated genomic DNA contamination, which may result in false methylation calls. As N3 blocks were observed in ENCFF710XQC but not its technical repeat ENCFF055UXZ, it also suggests that these 52 N3 blocks may not be relevant to biology but result from technical noise (Fig. 2b).

To evaluate the performance of "BCREval", we compared it with the existing BCR evaluator Bismark [8]. The results show that the methylation levels of $\mathrm{CpCs}$ in genome-wide (about $0.8 \%$ ) are in general higher than that in telomeres $(<$ $0.65 \%)$. And our algorithm has less recourse consumption (only 44 M Memory Usage), higher speed (30x faster) and comparable accuracy if not better (Table 1), which suggests the advantages of BCREval to be integrated into current WGBS pipelines. The major reason is that Bismark elucidates non-CpG methylation levels by aligning all reads to a bisulfite converted genome, which depends on aligner (for example Bowtie2) and is time/resource consuming.

\section{Discussion}

In a WGBS experiment, researchers implicitly assume that all unmethylated cytosines are converted into thymines, which unfortunately may not be the case. Based

Table 2 The data using in the manuscript

\begin{tabular}{|c|c|c|c|c|}
\hline Biosample Type & Library_ID & ENCODE_ID (FASTQ) & Strand & Biosample summary \\
\hline \multirow[t]{4}{*}{ Primary cell } & ENCLB587BLQ & ENCFF055UXZ & + & Homo sapiens skeletal muscle myoblast \\
\hline & ENCLB587BLQ & ENCFF764NTF & - & Homo sapiens skeletal muscle myoblast \\
\hline & ENCLB988SSO & ENCFF710XQC & + & Homo sapiens skeletal muscle myoblast \\
\hline & ENCLB988SSO & ENCFF331AID & - & Homo sapiens skeletal muscle myoblast \\
\hline \multirow[t]{6}{*}{ Cell line } & ENCLB542OXH & ENCFF336KJH & + & Homo sapiens K562 \\
\hline & ENCLB542OXH & ENCFF585HYM & - & Homo sapiens K562 \\
\hline & ENCLB890RFU & ENCFF211RZY & + & Homo sapiens HepG2 \\
\hline & ENCLB890RFU & ENCFF717MDZ & - & Homo sapiens HepG2 \\
\hline & ENCLB443JJF & ENCFF563QAT & + & Homo sapiens HepG2 \\
\hline & ENCLB443JJJF & ENCFF954LFD & - & Homo sapiens HepG2 \\
\hline \multirow[t]{4}{*}{ Stem cell } & ENCLB098BGY & ENCFF677BSB & + & Homo sapiens H1-hESC \\
\hline & ENCLB098BGY & ENCFF800KIP & - & Homo sapiens H1-hESC \\
\hline & ENCLB167QQW & ENCFF311PSV & + & Homo sapiens H1-hESC \\
\hline & ENCLB167QQW & ENCFF335TUD & - & Homo sapiens H1-hESC \\
\hline \multirow[t]{5}{*}{ Tissue } & ENCLB353RJB & ENCFF781BRM & + & Homo sapiens spleen male adult (37 years) \\
\hline & ENCLB353RJB & ENCFF535VCB & - & Homo sapiens spleen male adult (37 years) \\
\hline & ENCLB585SDT & ENCFF283GDL & + & Mus musculus C57BL/6 liver adult (54-61 day) \\
\hline & ENCLB506AYR & ENCFF978EJO & + & Mus musculus C57BL/6 liver adult (54-61 day) \\
\hline & ENCLB760KHX & ENCFF348XNA & + & Mus musculus C57BL/6 liver adult (54-61 day) \\
\hline
\end{tabular}




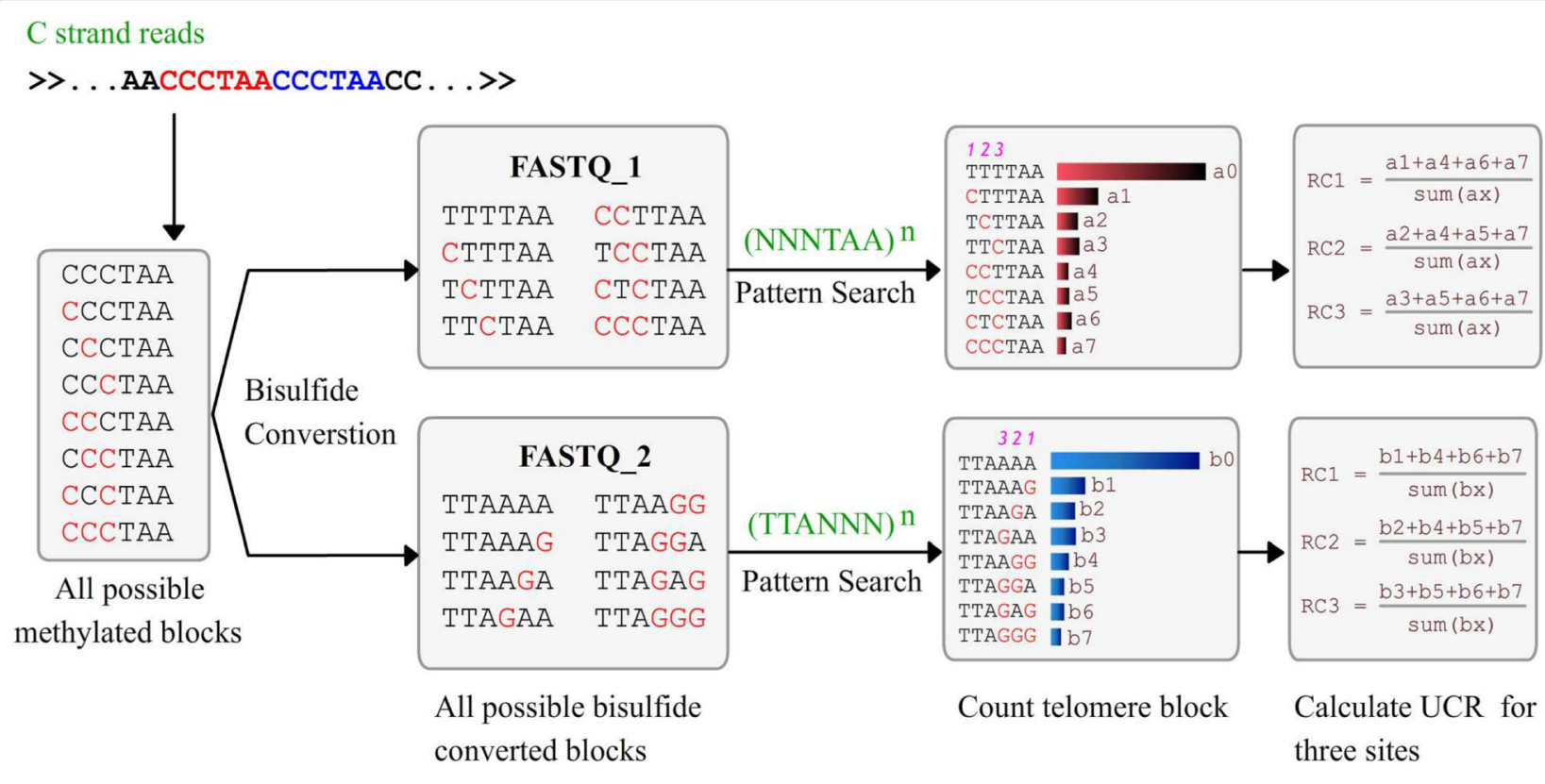

Fig. 4 The diagram about the procedure to calculate UCRs for three non-CpG cytosine sites using C-strand original reads. In the patterns, the N represents $\mathrm{G}$ or $\mathrm{C}$

on our knowledge current methods to monitor bisulfite conversion rate in WGBS experiments are time/resource consuming and may introduce false methylation calls. Here, we present a simple but robust method to QC and speculate BCR for WGBS experiments by using nonCpG sites in telomeric DNA as natural/internal controls.

We speculated 11 samples using our method and found: 1) Bisulfite conversion assay is really stable and usually achieves $>99.5 \%$ efficiency; 2) In most cases, the cytosine methylation at telomeres is a random processes and the methylation rates of telomere usually are very small $(<0.4 \%)$; 3$)$ No locational or sequence preferences are found in the three non-CpG sites; 4) Although it is impossible to distinguish random cytosine methylation from failure of bisulfite conversion in WGBS, our method have the potential to detect enzymatic DNA methylation by comparing the distribution of N1 and N2 blocks; 5) Our method has the ability to detect trace untreated genomic DNA contamination for QC purpose.

More importantly, comparing with existing BCR evaluator Bismark [8], BCREval has many advantages including faster processing, less recourse consumption and is easier integrated into presented WGBS pipelines. Although it should be kept in mind that telomeres, as native/inner control, might not necessarily have the same conversion properties and base nonCpG site methylation level as other genomic DNA, telomeric methylation state may still be a good indicator of baseline of no-CpG methylation and global DNA methylation dynamics.
Additionally, because all mammals share the same telomere DNA sequence, this method should be applicable to nonhuman mammalian samples without any modifications. it is also easy to modify program for other species with distinct telomere repeats by following the same principle of our method.

\section{Conclusion}

BCREval is a simple but robust method to speculates BCR for WGBS experiments to make sure it achieves acceptable level. It is much faster and more efficient than existing tools and can be easily integrated into current WGBS pipelines. A python script to implement BCREval is freely available at https://github.com/hqyone/BCR Evaluator.

\section{Methods}

All WGBS testing data (FASTQ files) were download form ENCODE database including 2 cell lines, 1 primary cell and 3 tissues (Table 2), All of them are pair-end WGBS data and have at least $20 \mathrm{M}$ reads with length above $100 \mathrm{bp}$. The home-made python script which implemented our algorithm can be download from here and has the ability to process multiple FASTQ files in a batch way. Detailed results can be found in a text output file.

The procedure of WGBS has been fully descripted other places [8]. Specifically, the way that telomeric DNA is processed in WGBS is illustrated in Fig. 3. It can be summarized as four steps: I. Double strand telomeric DNA fragments are composed by G-strand (5'-(TTAG 
GG) $\left.)_{n}-3^{\prime}\right)$ and C-strand (5'(CCCTAA $\left.)_{n}-3^{\prime}\right)$; II. dsDNA are denatured and become ssDNAs; III. Bisulfite treatment converts unmethylated cytosines in the $\mathrm{C}$ strand into thymines; IV. The polymerase chain reaction (PCR) library construction to form dsDNA; V. Next generation pair-end sequencing and data analysis. As the patterns of c-strand origin telomeric reads in two FASTQ files are known, it is easy to calculate UCR by comparing them with theoretical bisulfite covered sequences (Fig. 3 ). The details of our algorithm are shown in Fig. 4. The key step is searching two regular expressions ((NNNT AA) $n$ and (TTANNN) $n$ against FASTQ1 and FASTQ2 files respectively to find c-strand original reads, where $\mathrm{N}$ is $\mathrm{A}$ or $\mathrm{G}$ and the upper $\mathrm{n}$ is the minimal number of repeat units. The following step is counting all possible repeats and then calculating the unconverted ratios (UCRs) for all three non-CpG cytosines (Fig. 4). As both failure of bisulfite conversion and in vivo methylation at telomere are rare and independent, so UCRs can be mimic by the sum of the false conversion ratio (FCR) and methylated ratio (MR).

$$
\mathrm{UCR}=\mathrm{FCR}+\mathrm{MR}
$$

We classified six-bases telomeric blocks (TTAGGG) into four categories based on the number of unconverted cytosines (we named them as N0 N3 blocks). We considered the unconverted events as Bernoulli trials with certain possibility (UCR). So the percentage of N0 N3 telomeric blocks (R1 R3) should fit a binomial model and following formulas:

$$
\begin{aligned}
& C_{3}^{1} \times U C R \times(1-U C R)^{2}=R 1 \\
& C_{3}^{2} \times U C R^{2} \times(1-U C R)=R 2 \\
& C_{3}^{3} \times U C R^{3}=R 3
\end{aligned}
$$

As R1, R2 and R3 can be observed, it is easy to speculate UCR theoretically using these formulas. Actually, we only used the formula 2 to calculate UCRs because the sample size for $\mathrm{R} 1$ is much bigger than others.

All algorithm testing and comparisons were run at a DELL PowerEdge R730 server, with 128G RAM and two Xeon E5-2600 v3 processors with 18 cores. Bismark was downloaded from here (https://github.com/FelixKrueger/Bismark) and run following its manual with default settings. For each FSATQ file, 12 million reads are randomly extracted to save testing time.

\section{Supplementary information}

Supplementary information accompanies this paper at https://doi.org/10. 1186/s12859-019-3334-z.

Additional file 1: Figure S1. The location of N7 telomeric (TTAGGG) repeats in hg38 genome. Table $\mathbf{S 1}$. Detailed analysis results.

\section{Abbreviations}

BCR: Bisulfite conversion ratio; FCR: False conversion ratio; MR: Methylated ratio; PBAT: Post-bisulfite adapter tagging; PCR: Polymerase chain reaction; QC: Quality control; RRBS: Reduced representation bisulfite sequencing; UCR: Unconverted rate; WGBS: Whole genome shotgun bisulfite sequencing

\section{Acknowledgements}

We thank Dr. Qing Zhang (UCLA, USA) for suggestions and comments on the manuscript.

\section{Authors' contributions}

QYH and QZH: idea initiation, method development and data analysis; QYH, JZ and MZ: manuscript writing and data analysis; ZS, FW and YL: data collection; $\mathrm{XL}$ and $\mathrm{ZH}$ : preparing data and supplemental information. All authors read and approved the final manuscript.

\section{Funding}

This work was supported by the National Natural Science Foundation of China (31771445) and National Students' Platform for Innovation and Entrepreneurship Training Program (201810542018). QY H and ZS who were supported by these funding played major roles in the design of the study, data collection and writing the manuscript.

Availability of data and materials

A ready to use python script is available freely for all academic users at https://github.com/hqyone/BCR_Evaluator.

Ethics approval and consent to participate Not applicable.

\section{Consent for publication}

Not applicable.

\section{Competing interests}

The authors declare that they have no competing interests.

\section{Author details}

${ }^{1}$ School of Medicine, Hunan Normal University, Tongzipo Road 371, Changsha 410013, People's Republic of China. ${ }^{2}$ Verna and Marrs McLean Department of Biochemistry and Molecular Biology, Baylor College of Medicine One Baylor Plaza, Houston, TX 77-30, USA. ${ }^{3}$ The Affiliated Suzhou Hospital of Nanjing Medical University, Suzhou, People's Republic of China.

Received: 26 March 2019 Accepted: 19 December 2019

Published online: 31 January 2020

\section{References}

1. Miura F, Enomoto Y, Dairiki R, Ito T. Amplification-free whole-genome bisulfite sequencing by post-bisulfite adaptor tagging. Nucleic Acids Res. 2012:40(17):e136. https://doi.org/10.1093/nar/gks454.

2. Harris RA, Wang T, Coarfa $C$, et al. Comparison of sequencing-based methods to profile DNA methylation and identification of monoallelic epigenetic modifications. Nat Biotechnol. 2010;28(10):1097-105. https://doi. org/10.1038/nbt.1682.

3. Bernstein BE, Stamatoyannopoulos JA, Costello JF, et al. The NIH roadmap Epigenomics mapping Consortium. Nat Biotechnol. 2010;28(10):1045-8. https://doi.org/10.1038/nbt1010-1045.

4. Consortium TEP. An integrated encyclopedia of DNA elements in the human genome. Nature. 2012;489(7414):57-74. https://doi.org/10.1038/ nature 11247

5. Adams D, Altucci $L$, Antonarakis SE, et al. BLUEPRINT to decode the epigenetic signature written in blood. Nat Biotechnol. 2012;30(3):224-6. https://doi.org/10.1038/nbt.2153. 
6. The International Human Epigenome Consortium. 2016. http://ihecepigenomes.org/.

7. Krueger F, Kreck B, Franke A, Andrews SR. DNA methylome analysis using short bisulfite sequencing data. Nat Methods. 2012;9(2):145-51. https://doi. org/10.1038/nmeth.1828.

8. Krueger F, Andrews SR. Bismark: a flexible aligner and methylation caller for bisulfite-Seq applications. Bioinformatics. 2011;27(11):1571-2. https://doi.org/ 10.1093/bioinformatics/btr167.

\section{Publisher's Note}

Springer Nature remains neutral with regard to jurisdictional claims in published maps and institutional affiliations.

- fast, convenient online submission

- thorough peer review by experienced researchers in your field

- rapid publication on acceptance

- support for research data, including large and complex data types

- gold Open Access which fosters wider collaboration and increased citations

- maximum visibility for your research: over $100 \mathrm{M}$ website views per year

At $\mathrm{BMC}$, research is always in progress. 